\title{
EMOTIONAL LEARNING BASED INTELLIGENT CONTROLLERS FOR ROTOR FLUX ORIENTED CONTROL OF INDUCTION MOTOR
}

\author{
Rohollah Abdollahi * _ Reza Farhangi $^{* *}$ _ Ali Yarahmadi ${ }^{* * *}$
}

\begin{abstract}
This paper presents design and evaluation of a novel approach based on emotional learning to improve the speed control system of rotor flux oriented control of induction motor. The controller includes a neuro-fuzzy system with speed error and its derivative as inputs. A fuzzy critic evaluates the present situation, and provides the emotional signal (stress). The controller modifies its characteristics so that the critics stress is reduced. The comparative simulation results show that the proposed controller is more robust and hence found to be a suitable replacement of the conventional PI controller for the high performance industrial drive applications.
\end{abstract}

K e y w ords: induction motor, speed control, emotional learning, intelligent controller, PI controller

\section{INTRODUCTION}

AC motor drives are used in multitude of industrial and process applications requiring high performances. In high performance drive systems the motor speed should closely follow a specified reference trajectory regardless of any load disturbances, parameter variations and any model uncertainties. In order to achieve high performance, field oriented control of IM drive is employed [1]. However, the controller design of such system plays crucial role in the system performance. The decoupling characteristics of vector controlled induction motor are adversely affected by the parameters change in the motor.

The motor control issues are traditionally handled by fixed gain proportional integral (PI) and proportional integral derivative (PID) controllers. However, the fixed gain controllers are very sensitive to parameter variations, load disturbances, etc. So, the controller parameters have to be continually adapted. The problem can be solved by several adaptive control techniques such as model reference adaptive control (MRAC) [2], sliding mode control (SMC) [3], variable structure control (VSC) [4] and self tuning PI controllers [5], etc. The design of all of the above controllers depends on the exact system mathematical model. However, it is often difficult to develop an accurate system mathematical model due to unknown load variation, unknown and unavoidable parameter variations due to saturation, temperature variations and system disturbances. In order to overcome the above problems, recently the fuzzy logic controller (FLC) is being used for motor control purpose.

The mathematical tool for the FLC is the fuzzy set theory introduced by Zadeh [6]. As compared to the conventional PI, PID and their adaptive versions, the FLC has some advantages such as:

- it does not need any exact system mathematical model
- it can handle nonlinearity of arbitrary complexity

- it is based on the linguistic rules with IF-THEN general structure which is the basis of human logic.

However, the application of FLC has been facing some disadvantages during hardware and software implementation due to its high computational burden [7]. That is why so far the reported fuzzy logic works in motor drives [8-12] are mainly theoretical and based on either simulation or experimental results at very low speed operating conditions. With referring to above mentioned approaches it is clear up that fuzzy-logic control utilization to design speed control system of induction motor, rapidly increasing because of the good performance of this controller both in nonlinear and complex systems.

A fuzzy system includes a fuzzyfier of a deterministic input signal with a membership function, reasoning in a fuzzy rule set using a proper inference method, and defuzzyfier process to produce a deterministic output. Fuzzy rule base includes IF-THEN rules representing expert knowledge that makes decisions from input signals. This knowledge is provided by a control engineer who has performed extensive mathematical modeling, analysis, and development of control algorithms for power systems. Thus, fuzzy controllers work well as supervisory controllers in conditions such as severe nonlinearities, time varying parameters and plant uncertainties.

The proposed method in this study is the controlling model based on emotional processing in human beings brain that is latter method from above methods where the Critic gives rewards and punishments with respect to the states reached by the learner and is called Brain Emotional Learning Based Intelligent Controller (BEL$\mathrm{BIC})$. In real time control and decision systems, Emotional Learning is a powerful methodology due to its simplicity structure, low computational complexity, and independent from system model, online controlling and fast

\footnotetext{
* Department of Electrical Engineering, Shahab-e-Danesh Institute of Higher Education, Qom, Iran. rohollah.abdollahi@yahoo.com ** Department of Energy Engineering, Sharif University of Technology, Tehran, Iran, r_farhangi@energy.sharif.edu, *** Department of Electrical Engineering, Iran University of Science and Technology, Tehran, Iran, yarahmadi.ali@gmail.com
} 


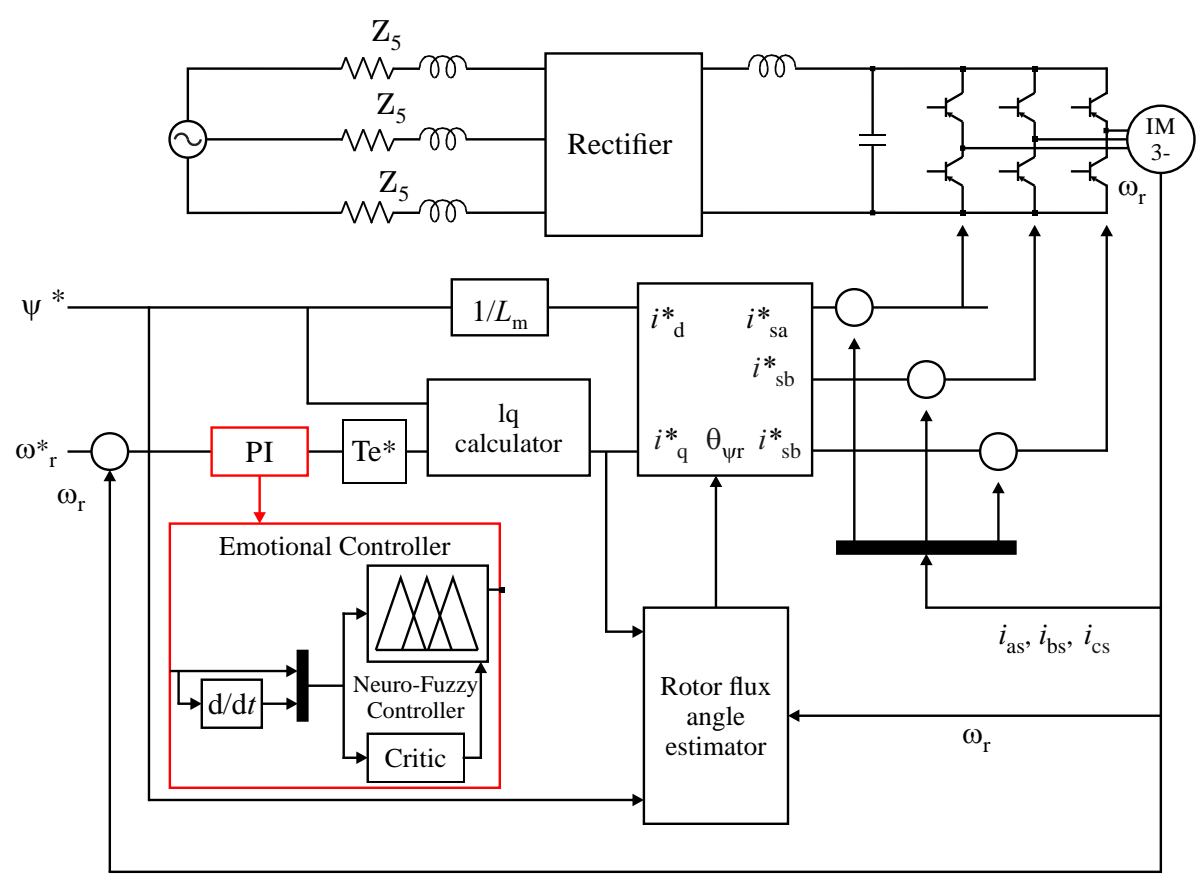

Fig. 1. The proposed emotional controller based IM drive

training. For these reasons, recently there is rising tend to intelligent controllers and BELBIC to use in different systems such as [14-17].

This novel approach applied to improve the speed control system of rotor flux oriented control of induction motor. The control system combined from a neuro-fuzzy controller and a fuzzy critic which evaluates the motor speed condition and then produces an appropriate signal to controller learning.

\section{ROTOR FLUX ORIENTED CONTROL OF INDUCTION MOTOR}

In this paper rotor flux oriented control will be applied to induction motor. The effects of magnetic saturation are neglected. Stator voltage equations can expressed (using complex notation) in the rotor flux reference frame as

$$
\begin{aligned}
V_{s \varphi_{r}}= & R_{s} i_{s \varphi_{r}}+L_{s} \frac{\mathrm{d} i_{s \varphi_{r}}}{\mathrm{~d} t}+L_{m} \frac{\mathrm{d} i_{r \varphi_{r}}}{\mathrm{~d} t}+ \\
& +j \omega_{m r} L_{s} i_{s \varphi_{r}}+j \omega_{m r} L_{m} i_{r \varphi_{r}}
\end{aligned}
$$

where $L_{m}$ is magnetizing inductance and $\omega_{m r}$ is rotor flux speed and $V_{s \varphi_{r}}$ can be express as

$$
V_{s \phi_{r}}=V_{s d}+j V_{s q}=\left(V_{s D}+V_{s Q}\right) e^{-j \theta_{r}}
$$

where $(d, q),(D, Q)$ and $r$ refer to rotor flux reference frame, stationary reference frame and flux rotor angel respectively. The rotor voltage equation will yield the following

$$
0=R_{r} i_{r \varphi_{r}}+\frac{\mathrm{d} \varphi_{r \varphi_{r}}}{\mathrm{~d} t}+j\left(\omega_{m r}-\omega_{r}\right) \phi_{r \varphi_{r}}
$$

where $\varphi_{r \varphi_{r}}$ rr is the rotor flux linkage in the rotor flux oriented reference frame and

$$
\varphi_{r \phi_{r}}=L_{m}\left|i_{m r}\right|
$$

By resolving into real and imaginary axis component, the simple equations are obtained

$$
T_{r} \frac{\mathrm{d}\left|i_{m r}\right|}{\mathrm{d} t}+\left|i_{m r}\right|=i_{s d}, \quad \omega_{m r}=\omega_{r}+\frac{i_{s q}}{\left|i_{m r}\right|}
$$

where $T_{r}=L_{r} / R_{r}$. The term $\left(i_{s q}\right) /\left(T_{r}\left|i_{m r}\right|\right)$ represents the angular rotor frequency $\omega_{s l},[1]$.

The conventional PI controller is one of the most common approaches for speed control in industrial electrical drives in general, because of its simplicity, and the clear relationship existing between its parameters and the system response specifications. The conventional PI controller fixed gains may perform well under some operating conditions but not all, because the involved processes are in general complex, time variant, with nonlinearity and model uncertainties. In order to improve the performances of the indirect vector control system, a novel approach based on emotional learning is being used to be the speed controller. The schematic diagram of the ELICbased indirect vector control of IM is shown in Fig. 1. The motor parameters are given in the Appendix.

\section{EMOTIONAL LEARNING}

There are three learning methods for neural networks characterized by the information source used for learning and classified with respect to the degree of information of the source. These learning methods are supervised learning, unsupervised learning, and reinforcement learning. 
Emotional learning is a type of reinforcement learning. It is done fuzzily and continuously in human being, in a way that the learning process is done through emotional signals. This signal is produced by the brain based on the persons behavior. Whenever the persons behavior is satisfactory, the stress is reduced in the person and no correction of the behavior is needed and as a result the value of the produced stress signal is small. If the persons behavior is not satisfactory, the stress is increased and as a result, the value of the stress signal is higher in order to improve the persons behavior.

Although, reinforcement learning and emotional learning have many similarities in training the controller systems, there also exist some differences in a way that the critic which is used in emotional learning has a continuous performance producing the learning signal in the range of $[-1,1]$. If the system operation is satisfactory, the value of this signal will be close to zero and if it is unsatisfactory its value is increased and based on the type of operation it will be close to 1 or -1 . But the critic which is used in reinforcement learning just analyzes success and failure in the system operation and based on this analyzing the learning signal is produced in order to train the controller ( 0 for failure in the system operation and 1 for success in the system operation).

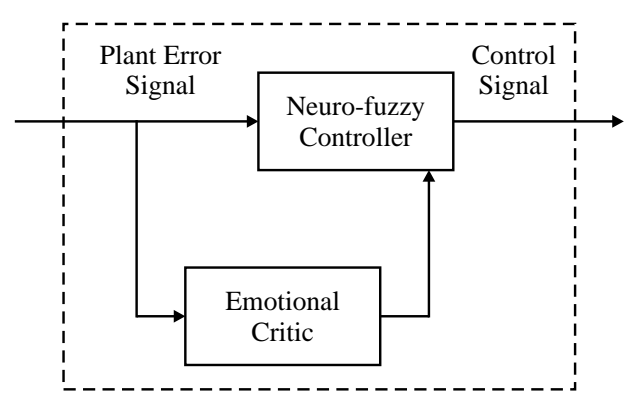

Fig. 2. Scheme of an emotional learning methodology

\section{EMOTIONAL LEARNING CONTROLLER}

Figure 2 shows the emotional learning controller structure which is used in this paper. The critic produces an emotional signal for the controller by analyzing the system performance. Controller amends its parameters based on this emotional signal and the current error in the system output in order to improve system performance. In this structure, because updating the controller parameters is based on the emotional signal, the system response is so dependent on the critic performance. Thus, the most important part in the control system is to design the critic. In this section, at first the neuro-fuzzy controller, then the operation of emotional critic, and finally the method of teaching the neuro-fuzzy controller is explained.

\subsection{Neuro-fuzzy controller}

Fuzzy systems are knowledge-based or rule-based systems [13]. The heart of a fuzzy system is a knowledge base consisting of the so-called fuzzy IF-THEN rules. A fuzzy IF-THEN rule is an IF-THEN statement in which some words are characterized by continuous membership functions. The starting point of constructing a fuzzy system is to obtain a collection of fuzzy IF-THEN rules from human experts or based on domain knowledge. The next step is to combine these rules into a single system. In fact the fuzzy system can be viewed as performing a real and nonlinear mapping from an input vector $X=\left[x_{1}, x_{2} \ldots x_{n}\right]^{T} \in R^{n}$ to an output vector $\tilde{y}=\tilde{f}(X) \in R^{m}\left({ }^{T}\right.$ denotes transposition; $n$ and $m$ are input and output vector dimensions). Different fuzzy systems use different principles for this combination. There are two types of fuzzy systems that are commonly used in the literature: Takagi-Sugeno-Kang (TSK), and fuzzy systems with fuzzyfier and defuzzyfier.

The model which is used here to design the neurofuzzy controller is of TSK type. Consider a multiple-input single-output (MISO) fuzzy system consisting of $N$ rules as follows:

- $R_{j}$ ( $j$-th rule): if $\left(x_{1}\right)$ is $\left.F_{j 1}\right)$ and $\left(x_{2}\right.$ is $\left.F_{j 1}\right)$ and $\left(x_{3}\right.$ is $\left.F_{j 3}\right)$ and $\ldots$ and $\left(x_{n}\right.$ is $\left.F_{j_{n}}\right)$ then $c_{j}=g_{j}(X)$, where $j=1,2, \ldots, N ; x_{i}(i=1,2, \ldots, n)$ are the input variables of the fuzzy system, $F_{j i}$ is characterized by its corresponding membership function $\mu F_{j i}\left(x_{i}\right), c_{j}$ is the consequence of the $j$-th rule and $g_{j}: \mathcal{R}^{n} \rightarrow \mathcal{R}^{m}$. Each rule $R_{j}$, can be viewed as a fuzzy implication by the inference engine.

- The antecedent fuzzy set (fuzzy Cartesian product) of each rule $F_{1} \times F_{2} \times \cdots \times F_{n}$ is quantified by the $t$-norm operator which may be defined as below, the min-operator or the product operator.

$$
\begin{aligned}
& \mu F_{1} \times \mu F_{2} \times \cdots \times \mu F_{n}\left(x_{1}, \ldots x_{n}\right)= \\
& =\left\{\begin{array}{l}
\min \left[\mu F_{1}\left(x_{1}\right) \times F_{2}\left(x_{2}\right) \times \cdots \times \mu F_{n}\left(x_{n}\right)\right] \\
\operatorname{or} \\
\mu F_{1}\left(x_{1}\right) \times F_{2}\left(x_{2}\right) \times \cdots \times \mu F_{n}\left(x_{n}\right)
\end{array}\right.
\end{aligned}
$$

- The defuzzification is then performed,

$$
\begin{gathered}
\tilde{y}=\tilde{f}(x)=\frac{\sum_{j=1}^{N} c \mu_{j}}{\sum_{j=1}^{N} \mu_{j}}, X=\left[x_{1}, \ldots, x_{n}\right] \in \mathcal{R}^{n} \\
\mu_{j}=\mu F_{1} \times \mu F_{2} \times \cdots \times \mu F_{n}\left(x_{1}, \ldots, x_{n}\right)
\end{gathered}
$$

where $\mu_{j}$ is the firing strength of the antecedent as a part of the $j$-th rule.

In TSK fuzzy systems, the consequent part of rules is given by

$$
c_{j}=a_{0 j}+\sum_{i=1}^{n} a_{i j} \cdot x_{i}
$$

where where $a_{0 j}$ and $a_{i j}$ are the coefficients that should be set at design stage or tuned during the corresponding learning procedure. Implementing a fuzzy inference 
system in the framework of an adaptive neural network results in a six layer network in which each layer serves as one part of the equivalent fuzzy system. Figure 3 shows a sample neuro-fuzzy system equivalent to a two-input and one-output TSK fuzzy inference system which has two linguistic labels for each input and therefore four rules in its rule base.

In the first layer, which is shown by I, the input is normalized to the range $[-1,1]$. In the second layer, which is shown by MF, by using the membership functions the input variables are transformed from real variables into linguistic variables. The third layer which is shown by c, multiplies the variables received from layer two and provides the antecedent part of the fuzzy rules $\mu_{F_{j 1}}\left(x_{1}\right) \times$ $\cdots \times \mu_{F_{j n}}\left(x_{n}\right)$. In the fourth layer, which is shown by $N$, the term $\mu_{j} / \sum_{j=1}^{n} \mu_{j}$ is calculated which expresses the ratio of firing strength in the $j$-th node to the sum of all firing strengths of the rules.

In the fifth layer, which is shown by T-S, by using the normalized data of the previous layer and the arranged TSK rules in this layer, the output of the above rules are calculated. Finally, the sixth layer is a defuzzyfier layer and the output is calculated based on (8).

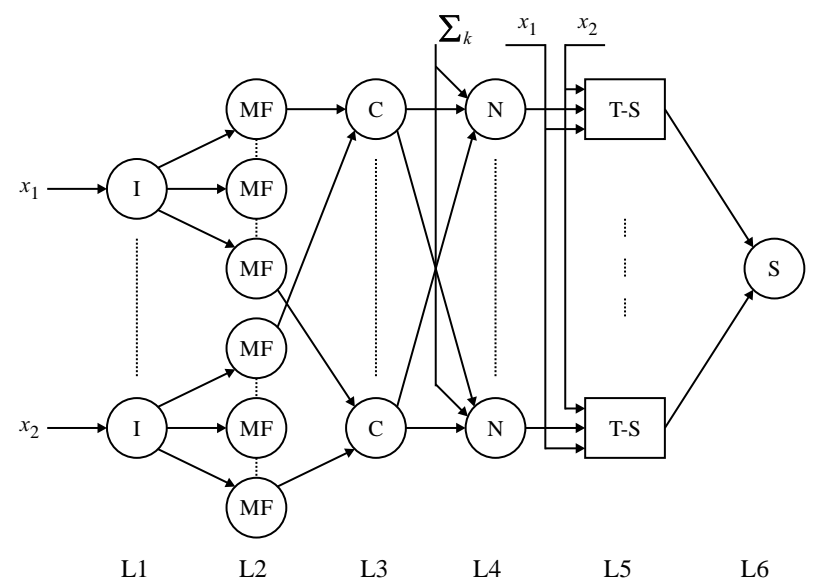

Fig. 3. Neuro-fuzzy structure equivalent with a MISO TSK fuzzy inference system with layers: L1 - input fuzzyfier, L2 - input linguistic, L3 - conjunction, L4 - normalizer, L5 - TSK rules and L6 - summation

\subsection{Emotional critic}

The performance of the critic is similar to the emotional section of human brain, in a way that it produces a learning signal in order to update the neuro-fuzzy controller weights by analyzing the system performance. This analysis is done by using the system error and its derivation signals. It means that position of system output and also the system behavior are effectual on the emotional signal. The critic is designed by implementing PD behavior via fuzzy systems. The critic which is designed by $\mathrm{PD}$ controller has a linear performance and it is not suggested to be used for non-linear systems. But the critic which is designed by neuro-fuzzy controller has a proper performance in non-linear systems. In this article, the expert fuzzy system model is used to design the critic.
Considering the fact that controller performance correction should lead to reduction of critic stress, the cost function is defined as follows

$$
E=\sum_{j=1}^{m} k_{j} \frac{r_{j}^{2}}{2}
$$

in which $r_{j}$ is the output emotional signal of critic $j$, $k_{j}$ is the weight of this signal, and $m$ is the number of system outputs which also defines the number of critics used in the system.

\subsection{Emotional learning}

As already mentioned, the main goal of emotional controller is to update the neuro-fuzzy controller parameters in order to reduce the critic stress based on cost function using steepest descent method

$$
E=\frac{1}{2} r^{2}, \quad \Delta \omega=-\eta \frac{\partial E}{\partial \omega}
$$

in which $\eta$ is the controller learning rate and $\omega$ is the tunable parameter of the controller. By using the chain rule in order to calculate equation XXX we will have

$$
\Delta \omega=-\eta \frac{\partial E}{\partial r} \frac{\partial r}{\partial y} \frac{\partial y}{\partial u} \frac{\partial u}{\partial \omega}
$$

in which $u$ is the control signal.

In the above equation $J$ is the system Jacobean. As the system input is increased its output will increase too, as a result the system Jacobean sign is positive in this system.

$$
\Delta \omega=-\eta r \frac{\partial r}{\partial y}(+1) \frac{\partial u}{\partial \omega}=-\eta r\left(\frac{\partial r}{\partial e} \frac{\partial e}{\partial y}\right) \frac{\partial u}{\partial \omega}
$$

Because the critic operation is fuzzy, $\frac{\partial r}{\partial e}$ value can be replaced by its symbol. Considering the fact that increase in the system error leads to increase in the stress, the sign of the above equation is positive. The system error is also calculated by using $e=y_{\text {ref }}-y$

$$
\Delta \omega=\eta r \frac{\partial u}{\partial \omega}
$$

In the introduced neuro-fuzzy controller, control signal $u$ of the previous sub-sections is calculated by combining (8) and (9) to get

$$
u=\frac{\sum_{j=1}^{N}\left(a_{0 j}+\sum_{i=1}^{n} a_{i j} x_{i}\right) \mu_{j}}{\sum_{j=1}^{n} \mu_{j}}
$$

Now based on (14) the controller parameters can be updated using

$$
\begin{gathered}
\Delta a_{0 j}=\eta r \frac{\partial u}{\partial a_{0 j}}=\eta r \frac{\mu_{j}}{\sum_{j=1}^{N} \mu_{j}} \\
\Delta a_{i j}=\eta r \frac{\partial u}{\partial a_{i j}}=\eta r x_{i} \frac{\mu_{j}}{\sum_{j=1}^{N} \mu_{j}}
\end{gathered}
$$




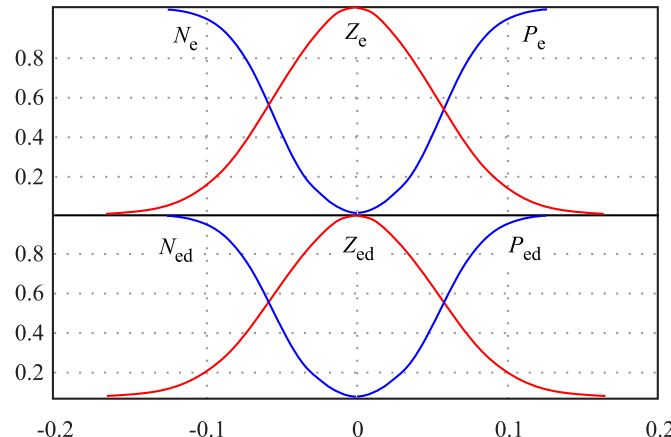

Fig. 4. The membership functions of the corresponding linguistic variables of the neuro-fuzzy Controller
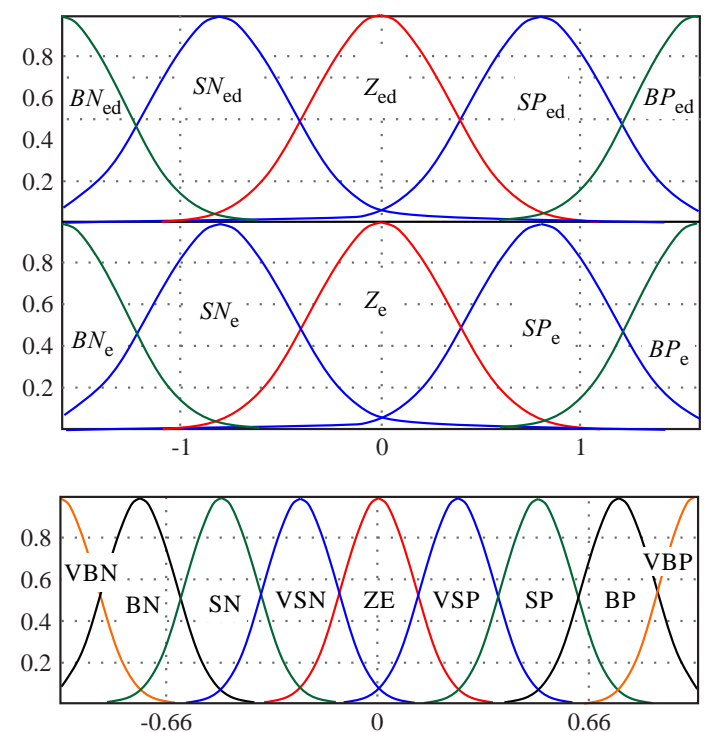

Fig. 5. Fuzzy critic membership functions: (a) - input membership functions and (b) - output membership functions

\section{THE PROPOSED SPEED CONTROL SYSTEM OF ROTOR FLUX ORIENTED CONTROL OF INDUCTION}

The structure of the designed emotional controller for speed control system of rotor flux oriented control of induction is shown in Fig.1. This structure is made of neurofuzzy controller and critic sections. The neuro-fuzzy controller section produces command signal in order to speed control system of rotor flux oriented control of induction.

As it was mentioned earlier the structure of this controller is of TSK type and speed error signal and its derivation are used as the inputs of this controller. In this controller three linguistic variables - Negative $(N)$, Positive $(P)$ and Zero $(Z)$ - are used in each input in order to tune the rules and according to this 9 rules are formed for the controller. Membership functions of linguistic variables are shown in Fig. 4.

It is obvious that sigmoid functions are used for variables $N$ and $P$, ie

$$
\mu F_{j i}\left(x_{i}\right)=\frac{1}{1+e^{-a_{j i}\left(x_{i}-c_{j i}\right)}}
$$

and Gaussian function, ie

$$
\mu F_{j i}\left(x_{i}\right)=-\left[\frac{x_{i}-c_{j i}}{\sigma_{j i}}\right]^{2}
$$

is used for variable $Z$. In the above equations $c_{i j}-$ is the center of function, $\sigma_{j i}$ - is the function variance and $a_{i j}$ - is the curve inflection function. The main sector in emotional controller is the critic. In this controller expert fuzzy model is used in order to design the critic. Speed error signal and its derivation are used as critic inputs in order to analyze system performance. Five linguistic variables are used for each of the above inputs; their membership functions are shown in Fig. 5(a). and Fig. 5(b). As it is seem in the figure, Gaussian function is used for variables $S P, S N$ and $Z$ and Sigmoid function is used for variables $B P$ and $B N$. According to the above linguistic variables, 25 different states can be defined in the critic and 25 different rules are tuned based on them in order to form the critic stress in the then part of these rules. The above rules are shown in Table 1 and also Fig. 5(c) shows the critic stress signal derived from these rules. For example if the speed error signal and its derivation are $B P$ (Big Positive), the system performance is unsatisfactory and the critic stress will be $V B P$; contrary to that if the speed error signal is SP (Small Positive) and its derivation is $S N$ (Small Negative) the system performance is satisfactory and the critic stress is also reduced.

Table 1. Critic fuzzy rule base

\begin{tabular}{ccccccc}
\hline Speed error & & BNe & SNe & Ze & SPe & BPe \\
\hline \multirow{5}{*}{ Speed error } & BNed & VBN & BN & SN & VSN & ZE \\
derivative & Zed & BN & SN & VSN & ZE & VSP \\
& SPed & VSN & ZE & VSP & SPP & BP \\
& BPed & ZE & VSP & SP & BP & VBP \\
\hline
\end{tabular}

Finally by applying the stress signal to the neuro-fuzzy controller, the controller parameters are tuned by using $\mathrm{SD}$ method in order to optimize system performance.

\section{RESULTS AND DISCUSSIONS}

Several tests were performed to evaluate the performance of the proposed ELIC based vector control of IM drive system simulated.

The speed control loop of the drive was also designed, simulated implemented with PI controller, in order to compare the performances to those obtained from the respective FLC based drive system. The speed responses are observed under different operating conditions such as sudden change in command speed, step change in load, 


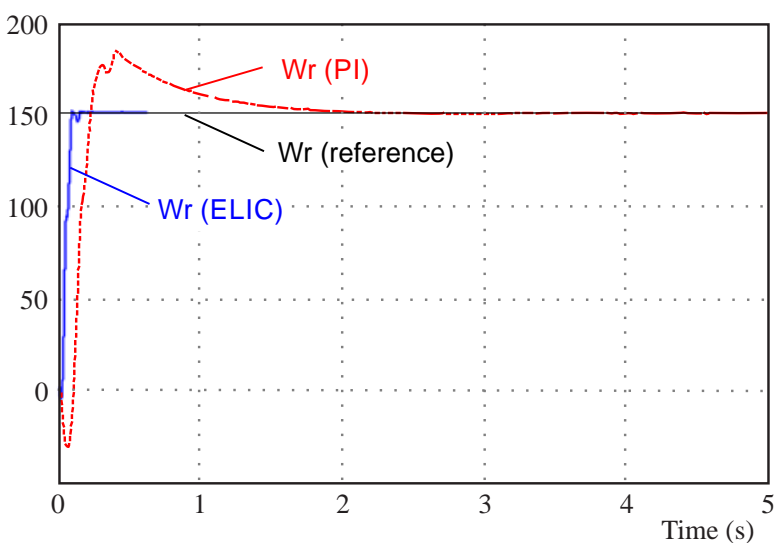

Fig. 6. Simulated starting responses of the drive with ELIC and PI

etc. Some sample results are presented in the following section.

The PI controller is tuned at rated conditions in order to make a fair comparison. Fig.6. show the simulated starting performance of the drive with PI and ELIC based drive systems, respectively. Although the PI controller is tuned to give optimum response at this rated condition, the emotional controller yielded better performances in terms of faster response time.

Figure 7 shows the speed responses of the drive system using PI and emotional controller, with step change in reference speed. It is evident from Fig. 7, that the proposed ELIC based IM drive system can follow the command speed without any overshoot and steady state error. So this intelligent controller is not affected by the sudden change of the command speed. Thus, a good tracking has been achieved for the ELIC. Whereas, the PI controller based drive system is affected with the sudden change in command speed

These figures also show that the ELIC based drive system can handle the sudden increase in command speed quickly without overshoot, undershoot and stead-state error, whereas the PI controller based drive system has steady-state error and the response is not as faster as compared to the ELIC. Thus, the proposed emotional controller based drive has been found superior to the conventional PI controller based system.

\section{CONCLUSION}

A novel Emotional learning based intelligent controllers to improve the speed control system of rotor flux oriented control of induction motor has been presented in this paper. The ELIC has been designed for speed control loop. The simulation has been carried out using SIMULINK Toolbox. The above controller is an intelligent controller of reinforcement learning type which uses a fuzzy critic in order to assess the system performance and tuning parameters of the controller. Since exact system parameters are not required in the implementation of the proposed controller, the perform-ance of the drive

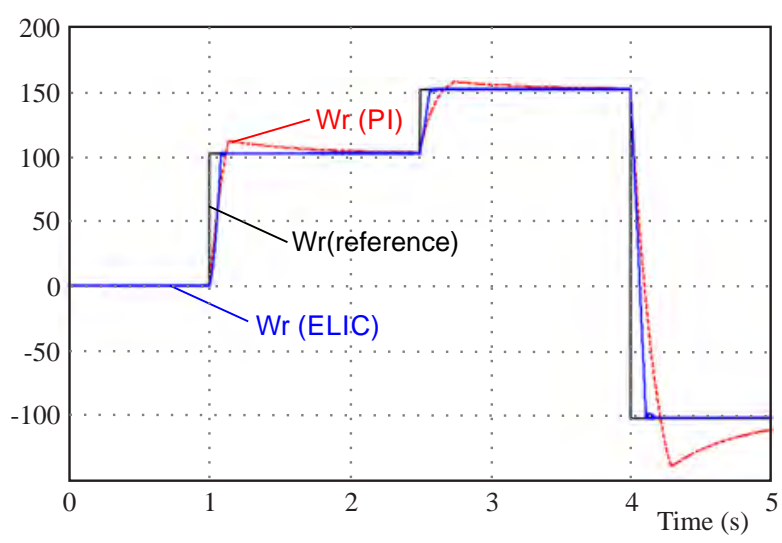

Fig. 7. Simulated speed responses of the drive due to step change of the reference speed ELIC and PI

system is robust, stable and insensitive to parameters and operating condition variations. In order to prove the superiority of the ELIC, a conventional PI controller based IM drive system has also been simulated implemented. It is concluded that the proposed Emotional learning based intelligent controllers has shown superior performances over the PI controller.

\section{Appendix}

Specifications of induction motor:

5 HP, 3-Phase, 4-Pole, Y-Connected, $460 \mathrm{~V}, 60 \mathrm{~Hz}$, $1800 \mathrm{rpm}$, squirrel cage induction type

$R_{s}=1.115 \Omega, R_{r}=1.083 \Omega, L_{s}=0.0059 \mathrm{H}, L=$ $0.0059 \mathrm{H}, L_{m}=0.2037 \mathrm{H}, J_{m}=0.01 \mathrm{kgm}^{2}, B_{m}=$ $0.02 \mathrm{Nms}$.

\section{REFERENCES}

[1] F. BLASCHKE: The Principle of Filed Orientation as Applied to The New Transvector Closed-Loop Control System for Rotating-Field Machines, Siemens Review 34 No. 3 (1972), 217-220.

[2] SUGIMOTO, H.-TAMAI, S. : Secondary Resistance Identification of an Induction Motor Applied Model Reference Adaptive System and its Characteristics, IEEE Trans. On Ind. Appl. 23 No. 2 (March/April 1987), 296-303.

[3] WON, CY.-BOSE, B. K.: An Induction Motor Servo System with Improved Sliding Mode Control, IEEE Conf. Rec. Of IECON'92 (1992), 60-66.

[4] CHERN, T. L.-WU, Y. C. : Design of Integral Variable Structure Controller and Application to Electrohydraulic Velocity Servo Systems, IEE Proceedings 138 No. 5 (September 1991), 439-444.

[5] HUNG, J. C.: Practical Industrial Control Techniques, IEEE Conf. Rec. of IECON'94 (1994), 7-14.

[6] ZADEH, L. A. : Fuzzy Sets, Information and Control 8 (1965), 338-353.

[7] BOlOGNANI, S.-ZIGLiOTTO, M.: Hardware and Software Effective Configurations for Multi-Input Fuzzy Logic Controllers, IEEE Trans, on Fuzzy Systems 6 No. 1 (Feb. 1998), 173-179.

[8] MIKI, I.-NAGAI, N.-NISHIYAMA, S.-YAMADA, T. : Vector Control of Induction Motor with Fuzzy PI Controller, IEEE/IAS Annual Meeting Conference Record (1991), 341-346. 
[9] MIR, []S. A.-ZINGER, D. S. : Fuzzy Controller for Inverter Fed Induction Machines, IEEE/IAS Annual Meeting Conference Record (1992), 464-471.

[10] TANG, Y.-XU, L.: Fuzzy Logic Application for Intelligent Control of a Variable Speed Drive, IEEE Trans, on Energy Conversion 9 No. 4 (Dec. 1994), 679-685.

[11] CeRruto, E.-CONSOli, A.-RACITI, A.-TESTA, A.: Fuzzy Adaptive Vector Control of Induction Motor Drives, IEEE Trans, on Power Electronics 12 No. 6 (Nov. 1997), 1028-1039.

[12] FAKHRAZARI, A.-BOROUSHAKI, M. : Adaptive Critic-base Neurofuzzy Controller for the Steam Generator Water Level, IEEE Transactions On Nuclear Science 55 No. 3 (JUNE 2008), 1678-1685.

[13] JAFARZADEH, S.-MIRHEIDARI, R.-MOTLAGH, M. R. J.-BARKHORDARI, M. : Intelligent Autopilot Control Design for a 2-DOF Helicopter Model, International Journal of Computers, Communications \& Control 3 (2008), 337-342.

[14] KHORRAMABADI, S. S.-BOROUSHAKI, M.-LUCAS, C. Emotional learning based intelligent controller for a PWR nuclear reactor core during load following operation, Annals of Nuclear Energy 35 No. 2 (2008), 2051-2058.

[15] GHOliPOUR, A.-LUCAS, C.-SHAhMirzadI, D.: Predicting Geomagnetic Activity Index by Brain Emotional Learning, WSEAS Transactions on Systems 3 No. 1 (2004), 296-299.

[16] JAFARZADEH, S.-MIRHEIDARI, R.-JAHED, M.-BARKHORDARI, M. : Designing PID and BELBIC Controllers in Path Tracking Problem", International Journal of Computers, Communications and Control 3 (2008), 343-348.
Received 7 May 2013

Rohollah Abdollahi was born in Qom, Iran, in 1983. He received the BSc degree in electrical engineering from Buali Sina University, Hamedan, Iran, in 2007 and the MSc degree in electrical engineering (power electronics and electrical machines) from Iran University of Science and Technology, Tehran, Iran, in 2011. He is faculty the Electrical Engineering Department, Shahab-e-Danesh Institute of Higher Education, Qom, Iran. He is the author of more than 20 journal and 7 conference papers and 8 Patents. His principle research interests are: Power electronics, Power quality, and Electrical machines. Mr. Abdollahi is a member of Iran's National Elite Foundation and a member of Iranian organization for engineering order of building and a member of the Iranian association of electrical and Electronics Engineers

Reza Farhangi was born in Qom, Iran, in 1985. He received the BSc degree in electrical engineering from Buali Sina University, Hamedan, Iran, in 2007 and the MSc from Iran University of Science and Technology, Tehran, Iran, in 2011.

Ali Yarahmadi was born in Arak, Iran, in 1984. He received the BSc degree in electrical engineering from Buali Sina University, Hamedan, Iran, in 2007 and the MSc degree in electrical engineering (power electronics and electrical machines) from Iran University of Science and Technology, Tehran, Iran, in 2011.

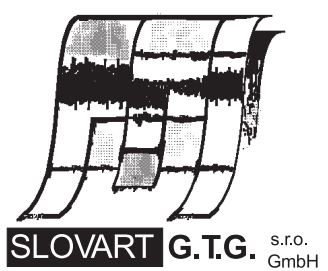

EXPORT - I MPORT
EXPORT - I M P OR T

of periodicals and of non-periodically printed matters, books and CD-ROMs

Krupinská 4 PO BOX 152, 85299 Bratislava 5, Slovakia tel: ++421 263839 472-3, fax: ++421263839485 info@slovart-gtg.sk; http://www.slovart-gtg.sk

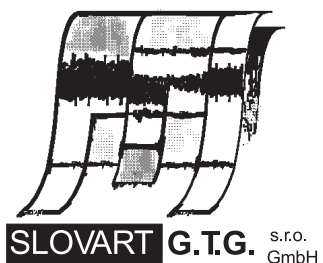

EXPORT - IMPORT 